\title{
MODELAGENS ANALÍTICAS APLICADAS AO CONTROLE DE CUSTOS NA GERÊNCIA DA PRODUÇÃO E OPERAÇÕES
}

Rodrigo Roratto ${ }^{1}$

\section{RESUMO}

A gestão de custos em processos de produção é bastante complexa e requer o emprego de adequadas técnicas de análise e controle. Por isso, o presente artigo tem como objetivo geral descrever modelagens analíticas que possam ser adotadas em diferentes situações por quaisquer organizações, a fim de controlar custos no gerenciamento de processos produtivos. Para isso, após uma análise teórica fundamentada em algumas das principais técnicas de pesquisa operacional, construiu-se a modelagem conforme cada situação hipotética preestabelecida, que poderia ser vivenciada por uma organização. Primeiramente, foi apresentado um estudo de caso em que o modelo construído foi baseado na rede PERT/CPM, para a análise do cronograma e orçamento de um projeto. Já em outra situação, foi elaborada uma modelagem matemática, que empregou a técnica de programação linear para o encontro do objetivo de redução de custos num projeto específico. Assim, tais modelagens apresentadas podem ser aplicadas de acordo com a situação objetivada pela organização na gestão de custos de produção, bastando apenas buscar as variáveis e modelar o estudo de acordo com o método que se quer aplicar para análise.

Palavras-Chave: Gestão de projetos. Planejamento de custos. Pesquisa operacional. 
Atualmente, técnicas de gerenciamento de projetos são utilizadas por empresas dos mais diversos ramos de atividade econômica e têm sido de fundamental importância para transformar o planejamento em resultados, otimizar a alocação de recursos, diminuir as surpresas e proporcionar maior lucratividade ao processo de gestão.

Porém, em alguns casos, fica difícil mensurar quais projetos agregam valor para o desempenho organizacional, visto que muitas organizações não possuem fins lucrativos ou a prioridade de uma empresa, que, em dado momento, pode estar na contenção de custos em determinada atividade de produção. Isto é, nesses casos, o que interessa é a elaboração de projetos que aperfeiçoem processos e reduzam o desperdício dos recursos (minimização de custos). Nesse momento, torna-se mais difícil decidir qual a melhor metodologia ou modelagem a ser aplicada, haja vista a pouca utilização de sofisticadas técnicas de controle de custos durante o gerenciamento de projetos.
Diante dessa problemática, o presente artigo tem como objetivo geral descrever modelos matemáticos baseados em técnicas de Pesquisa Operacional que possam ser adotados em diferentes situações por quaisquer organizações, a fim de planejar (ou minimizar) custos no gerenciamento de projetos produtivos. Para isso, após uma análise teórica sobre o assunto, construiu-se os modelos conforme cada situação hipotética preestabelecida. Como justificativa para a realização de uma pesquisa desta natureza, além da busca de subsídios para a redução de incertezas na tomada de decisões nesse tipo de estudo, estaria na necessidade de definir qual técnica voltada à redução de custos pode ser a mais apropriada para cada situação planejada por uma organização.

Assim, o artigo inicia-se por esta introdução e segue com o item Pesquisa Operacional e a Gestão de Custos em Projetos, Metodologia, Modelagem Analítica,Conclusão e Referências.

\section{PESQUISA OPERACIONAL E O PLANEJAMENTO DE CUSTOS NA PRODUÇÃO E OPERAÇÕES}

Conforme Pormpermayer e Lima (2002), o lucro é a seiva que nutre e robustece o patrimônio das entidades. Entretanto, segundo eles, o lucro não é apenas uma simples verificação de uma desigualdade. Se o valor da receita é maior do que o valor da despesa, o resultado é lucro $(\mathrm{R}>\mathrm{D}=\mathrm{L})$. O lucro é a consequência da soma parcial ou total de qualidade e produtividade, de emoção e razão, de intuição e lógica, de capacidade empresarial e trabalho, de fé e persistência, de firmeza e equilíbrio, de vontade e garra. Portanto, ainda de acordo com os autores, conclui-se que o lucro deixou de ser atributo da receita, das vendas, mas, 
sim, função resultante dos custos incorridos, de tal modo que, almejar lucro, é conter custos. Lucros e custos são grandezas inversamente proporcionais, isto é, o lucro será máximo se o custo for mínimo.

O gerenciamento dos custos do projeto inclui os processos envolvidos em estimativas, orçamentos e controle dos custos, de modo que o projeto possa ser terminado dentro do orçamento aprovado (PMBOK, 2012). Diante disso, o processo de controle de custos tem por objetivos fundamentais (PMBOK, 2012; SOTILLE, 2003; DINSMORE, 2003):

a) monitorar o desempenho do custo para detectar suas variações;

b) assegurar que todas as mudanças aprovadas estão registradas no baseline de custo;

c) impedir que mudanças não aprovadas ou incorretas sejam incluídas no baseline do custo;

d) informar as mudanças autorizadas a todas as partes envolvidas;

e) preservar a saúde financeira do projeto, mantendo os custos dentro de limites aceitáveis.

O conhecimento, a aquisição de dados e medição precisa dos custos de mão de obra, de equipamentos e de materiais são fundamentais para avaliação correta da produtividade do projeto, sendo que comparação dos custos planejados com os reais revelará se o projeto está evoluindo conforme planejado (PEREZ FILHO,2006). Portanto, segundo Perez Filho (2006), a identificação das causas dos desvios, tanto positivos quanto negativos, ensejará ao gerente de projeto tomar as medidas corretivas e/ou preventivas necessárias, visando restabelecer as condições originalmente estabelecidas ou, até mesmo, melhorá-las. Logo, o eficaz processo de gestão de custos passa, necessariamente, pela eficiência e eficácia das atividades de planejamento, execução e controle.

É importante ressaltar que, na fase de planejamento, a Pesquisa Operacional poderia contribuir muito nesse sentido, por meio de algumas técnicas sofisticadas voltadas à gestão de custos. Para Shamblin e Stevens Jr (1979), a Pesquisa Operacional (PO) é “[...] um método científico de tomada de decisão”. Ela inicia descrevendo um sistema por intermédio de um modelo e depois lida com este modelo para levantar o melhor modo de operar o sistema.

De acordo com Lachtermacher (2009), a PO com um enfoque mais clássico é definida como a arte de aplicar técnicas de modelagem a problemas de decisão, por meio de métodos matemáticos e estatísticos, buscando encontrar a solução ótima de maneira sistêmica.Já em um enfoque atual, a PO leva em consideração as interações com o ambiente interno e externo para a formulação da modelagem de um problema. Ackoff e Sasieni (1974) também mostram a forma de equações que os modelos de PO assumem. Para eles, esta forma é de estrutura básica e muito simples:

$$
\mathrm{Z}=f\left(\mathrm{Xi}, \mathrm{Yj}_{\mathrm{j}}\right)
$$

Onde:

$Z$ - é a utilidade ou valor do desempenho (performance) do sistema (será chamada de função objetivo);

$\mathrm{X} \mathrm{i}-$ são as variáveis que podem ser controladas; $Y j$ - são as variáveis (ou constantes) que não podem ser controladas, mas que afetam $Z$; e

$f$-é o relacionamento entre $\mathrm{Z}, \mathrm{Xi}, \mathrm{Yj}$. 
Segundo Montevechi (2006), após o modelo matemático ser construído, pode ser necessário simplificá-lo, para ser tratado analiticamente. Segundo Williams (1993), o termo modelo é normalmente utilizado para caracterizar estruturas que representam feições e objetos ou cenários, que podem ser dirigidos à análise de custos.

\section{METODOLOGIA}

Em relação aos procedimentos técnicos, a pesquisa é caracterizada como um estudo bibliográfico, que visa recorrer à literatura pertinente para uma análise de conceitos e trabalhos anteriores que possam servir de embasamento a aplicações práticas, por meio do levantamento de técnicas de $\mathrm{PO}$ referentes ao planejamento de projetos produtivos. Além disso, quanto aos objetivos, é considerada uma pesquisa do tipo descritiva, que, segundo Vergara (2006), permite a apresentação das características de um evento, sem a necessidade de explicar os fenômenos que descreve, mesmo que possa embasar tal explicação.

Quanto à abordagem, é considerado um estudo de caráter quantitativo, uma vez que expõe delineamentos de modelagem matemática, baseados na rede PERT/CPM (ProgramEvaluation and Review Technique / Critical Path Method), oriunda da teoria dos grafos e da programação linear (da programação matemática), que permite o processo de planejamento das atividades de produção e dos recursos tomados como base nos estudos de caso levantados. Conforme Terence e Escrivão Filho (2006), uma pesquisa de tipologia quantitativa possibilita mensurar as variáveis de estudo no intuito de proporcionar maior precisão aos seus resultados.

É importante ressaltar que a construção matemática está limitada à apresentação de apenas duas técnicas de PO, escolhidas por serem de ampla utilização pelo meio acadêmico, bem como por permitirem uma aplicação que possa satisfazer a tomada de decisão em amplo espectro de projetos, conforme cada situação vivenciada (ou necessitada) por inúmeras organizações, tanto do setor público quanto privado. Destarte, esta pesquisa apresenta, em sua estrutura de resultados, o embasamento teórico de cada modelagem de $\mathrm{PO}$ adotada e sua respectiva aplicação prática, onde os exemplos utilizados foram extraídos de trabalhos voltados a esse tipo de aplicação matemática.

\section{MODELAGEM ANALÍTICA}

\subsection{Modelagem pelo método PERT/CPM}

Segundo Shimizu (1984), esse método ébaseado na teoria dos grafos, sendo aplicável em projetos cujos prazos de execução de cada atividade sejam estabelecidos considerando-se um certo nível de incerteza, que acaba por gerar estimativas probabilísticas para a análise de viabilidade. O Método da rede PERT calcula a média ponderada de três cenários de tomada de decisão (geralmente nos casos otimista, pessimista e mais provável). Já o CPM busca levantar o caminho crítico de uma sequência de atividades 
produtivas, que se configura no conjunto de atividades cuja duração impacta no tempo de duração de um processo ou projeto. Assim sendo, o PERT possui caráter probabilístico e o CPM é de cunho determinístico.
De acordo com Bachega e Antonialli (2004), existem alguns conceitos básicos necessários para se trabalhar com o Método PERT/CPM, tais como apresentados no Quadro.

Quadro 1: Conceitos sobre PERT/CPM

\begin{tabular}{|c|c|}
\hline Item & o \\
\hline Evento & $\begin{array}{l}\text { São pontos no tempo quando se toma uma decisão (representados por círculos, } \\
\text { quadrados etc. numa rede PERT). Nestes pontos, não é necessário trabalho, já } \\
\text { que são considerados os objetivos. }\end{array}$ \\
\hline Atividade & $\begin{array}{l}\text { É a execução efetiva de uma tarefa, na qual se consome recursos e tempo } \\
\text { (geralmente representadas por setas que interligam os eventos). As atividades } \\
\text { podem ser: } \\
\text { a) paralelas, ou seja, atividades que ocorrem paralelamente entre dois eventos; } \\
\text { b) dependente, ou seja, para que a atividade seja realizada, esta depende do } \\
\text { cumprimento de outra (s); } \\
\text { c) independente, ou seja, não depende integralmente das atividades que chegam } \\
\text { ao nó de onde ela partiu; } \\
\text { d) fantasmas (ou dummy), pode existir uma única atividade entre dois eventos } \\
\text { sucessivos e, para evitar este problema, há o artifício de se usarem atividades } \\
\text { fantasmas que não consomem tempo nem recursos; } \\
\text { e) condicionantes, ou seja, atividades que podem ser executadas somente sob } \\
\text { determinadas condições ou tempo preestabelecido. }\end{array}$ \\
\hline $\begin{array}{l}\text { Estimativas de } \\
\text { Tempo da Rede } \\
\text { Pert }\end{array}$ & $\begin{array}{l}\text { a) Tempo otimista: menor tempo possível no qual a atividade possa ser executa- } \\
\text { da, ou seja, o tempo necessário para completar o trabalho, caso tudo ocorra } \\
\text { melhor do que o esperado. } \\
\text { b) Tempo mais provável: estimativa de tempo mais exata possível. Seria, então, } \\
\text { o tempo gasto se tudo ocorrer satisfatoriamente. } \\
\text { c) Tempo pessimista: máximo de tempo necessário para a execução da atividade. } \\
\text { Nesta estimativa de tempo, seriam considerados os fatores adversos. } \\
\text { d) Tempo mais cedo: maior tempo necessário para que o evento seja atingido, } \\
\text { desconsiderando imprevistos e atrasos nas atividades antecedentes, ou seja, } \\
\text { a soma do tempo inicial do projeto com o somatório dos tempos anteriores } \\
\text { ao evento analisado. } \\
\text { e) Tempo mais tarde: menor tempo necessário para realização de um evento, ou } \\
\text { seja, a diferença entre o tempo total de conclusão do projeto com o somatório } \\
\text { dos tempos posteriores ao evento analisado. } \\
\text { f) Tempo de conclusão: tempo total de conclusão do projeto ou da obrigação } \\
\text { contratual. } \\
\text { g) Folga: medida de excesso de tempo disponível para a realização do evento, } \\
\text { obtida pela diferença entre o último tempo permissível e o tempo esperado. } \\
\text { h) Caminho crítico: é o caminho na rede PERT que possui folga igual a zero } \\
\text { (que não pode sofrer atraso) e que determina o tempo esperado de execução } \\
\text { do projeto. }\end{array}$ \\
\hline
\end{tabular}


É definida com a relação entre duas atividades contíguas, de maneira que uma atividade, dependente somente, possa ser iniciada quando a imediatamente precedente estiver conclusa.

Fonte: adaptado de Bachega e Antonialli (2004)

\subsubsection{Exemplo Prático}

Neste tópico, será apresentado um estudo de caso relativo a um projeto de produto correspondente, nesse caso, ao projeto de implantação de um novo sistema de tecnologia da informação (TI) junto a uma empresa que deseja automatizar seus processos de gestão. Para tal, foram dadas as atividades necessárias para a execução do respectivo projeto e suas perspectivas de tempo em diferentes cenários, o custo previsto para cada estimativa (quanto a organização teria que desembolsar na execução do projeto em cada cenário), o tempo máximo fixado para a conclusão e a disponibilidade orçamentária (limitação financeira) fixada para o custeio do projeto, de acordo com o Quadro 2.

Quadro 2: Visão inicial do projeto de um sistema de TI e suas restrições, dentro dos cenários propostos pela organização

\begin{tabular}{|c|c|c|c|c|}
\hline \multirow[b]{2}{*}{ Atividades } & \multirow[b]{2}{*}{$\begin{array}{l}\text { Sucessor } \\
\text { imediato }\end{array}$} & \multicolumn{3}{|c|}{$\begin{array}{c}\text { Estimativas de tempo } \\
\text { (em mês) }\end{array}$} \\
\hline & & $\begin{array}{c}\text { (O) } \\
\text { Otimista }\end{array}$ & $\begin{array}{c}\text { (M) } \\
\text { Mais } \\
\text { provável }\end{array}$ & $\begin{array}{c}\text { (P) } \\
\text { Pessi- } \\
\text { mista }\end{array}$ \\
\hline $\begin{array}{l}\text { A. Análise do plano estratégico institucional e } \\
\text { discussão sobre suas metas e objetivos }\end{array}$ & B & 1,5 & 2 & 3 \\
\hline $\begin{array}{l}\text { B. Identificação dos usuários internos e externos, } \\
\text { bem como suas exigências e necessidades }\end{array}$ & $\mathrm{C}$ & 1,5 & 2,5 & 3 \\
\hline C. Identificação dos dados que devem estar na base & $\mathrm{D}, \mathrm{E}$ & 1 & 1,5 & 2 \\
\hline D. Estruturação e delineamento da base de dados & $\mathrm{F}$ & 1,5 & 2 & 2,5 \\
\hline E. Mapeamento do fluxo de processos & $\mathrm{H}$ & 0,2 & 0,5 & 1,5 \\
\hline $\begin{array}{l}\text { F. Armazenagem dos dados na base (suprimento do } \\
\text { banco de dados) }\end{array}$ & G & 2,5 & 3,5 & 4 \\
\hline
\end{tabular}




\begin{tabular}{|c|c|c|c|c|}
\hline \multirow[b]{2}{*}{ Atividades } & \multirow[b]{2}{*}{$\begin{array}{l}\text { Sucessor } \\
\text { imediato }\end{array}$} & \multicolumn{3}{|c|}{$\begin{array}{l}\text { Estimativas de tempo } \\
\text { (em mês) }\end{array}$} \\
\hline & & $\begin{array}{c}\text { (O) } \\
\text { Otimista }\end{array}$ & $\begin{array}{c}\text { (M) } \\
\text { Mais } \\
\text { provável }\end{array}$ & $\begin{array}{c}(\mathrm{P}) \\
\text { Pessi- } \\
\text { mista }\end{array}$ \\
\hline G. Modelagem dos dados armazenados & I & 1 & 1,5 & 2 \\
\hline H. Padronização de processos e tarefas & $\mathrm{L}$ & 1 & 2 & 3 \\
\hline $\begin{array}{l}\text { L. Implementação de medidas de controle e } \\
\text { segurança da informação }\end{array}$ & $\mathrm{N}$ & 1 & 1,5 & 2 \\
\hline M. Pesquisa de satisfação de usuários (feedback) & $\mathrm{N}$ & 1 & 2 & 2,5 \\
\hline N. Ajustes conforme necessidades dos usuários & - & 0,5 & 1 & 1,5 \\
\hline \multicolumn{2}{|c|}{ CUSTO TOTAL PREVISTO EM CADA ESTIMATIVA (R\$) } & $1.698,00$ & $1.857,00$ & $2.130,00$ \\
\hline \multicolumn{4}{|c|}{ TEMPO TOTAL FIXADO PARA A EXECUÇÃO DO PROJETO (Meses) } & 18 \\
\hline DISPONIBILIDADE ORÇAMENTÁRIA (R\$) & & & & $1.900,00$ \\
\hline
\end{tabular}

Fonte: dos autores (2017)

A partir do quadro anterior, é possível elaborar a rede PERT/CPM do projeto com a seguinte distribuição de eventos e atividades.

Figura 1: Rede PERT/CPM do projeto

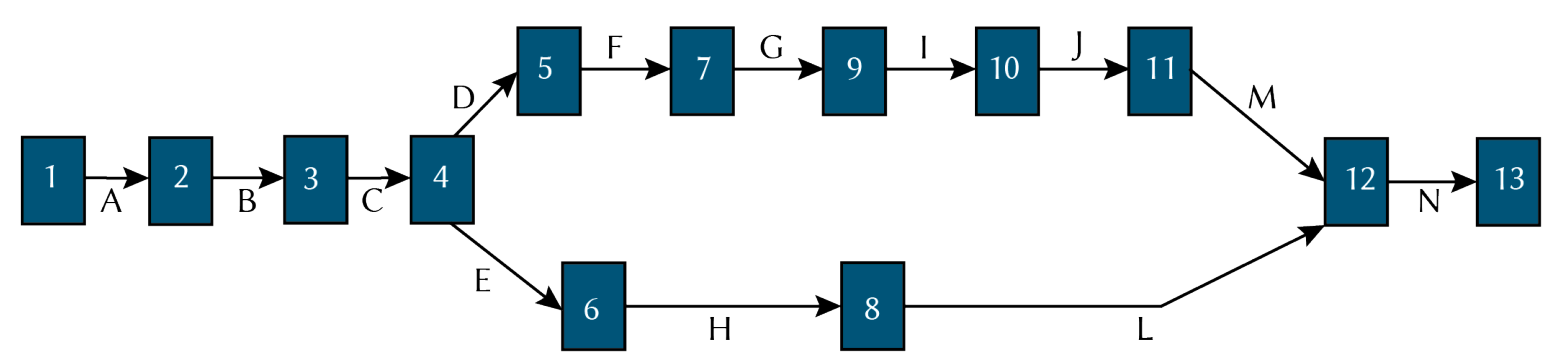

Fonte: dos autores (2017) 
Ao analisara rede eo Quadro1, com os dados iniciais, aplica-se as fórmulas matemáticas da rede PERT/CPM para se obter, respectivamente:

a) o tempo esperado de cada atividade $\left(\mathbf{T}_{\mathbf{e}}\right.$ $=\mathrm{O}+4 \mathrm{M}+\mathrm{P} / 6$ );

b) a variância de cada atividade $(\mathbf{V}=[(\mathbf{P}$ $\left.-0) / 6]^{2}\right)$

c) o tempo mais cedo de cada evento $\left(T_{\text {+cedo }}=T_{\text {inicial do projeto }}+\Sigma T_{\text {anteriores ao evento }}\right)$; d) o tempo mais tarde do evento $\left(\mathbf{T}_{+ \text {tarde }}=\right.$ $\left.\mathrm{T}_{\text {final do projeto }}-\boldsymbol{\Sigma} \mathrm{T}_{\text {posteriores ao evento }}\right)$;

e) a folga de cada evento $\left(\mathbf{T}_{+ \text {tarde }}-\mathbf{T}_{+ \text {cedo }}\right)$, que determinará quais eventos serão críticos (com folga nula) e que não poderão sofrer atraso.

No quadro 2, são aplicadas as fórmulas para a solução do problema.

Quadro 3: Análise dos meses de execução do projeto por meio da técnica PERT/CPM

\begin{tabular}{|c|c|c|c|c|c|c|c|}
\hline $\begin{array}{c}\text { Tempo } \\
\text { Atividade }\end{array}$ & $\begin{array}{c}\text { Esperado } \\
\qquad\left(T_{e}\right)\end{array}$ & Variância & $\begin{array}{l}\text { Tempo } \\
\text { Evento }\end{array}$ & $\begin{array}{l}\text { Mais cedo } \\
\qquad\left(T_{+ \text {cedo }}\right)\end{array}$ & $\begin{array}{l}\text { Mais } \\
\text { tarde } \\
\left(\mathrm{T}_{+ \text {tarde }}\right)\end{array}$ & $\begin{array}{c}\text { Folga } \\
\left(\mathrm{T}_{\text {+tarde }}-\right. \\
\left.\mathrm{T}_{\text {+cedo }}\right)\end{array}$ & $\begin{array}{l}\text { Evento } \\
\text { crítico? }\end{array}$ \\
\hline A & 2,1 & 0,063 & 1 & 0 & 0 & 0 & Sim \\
\hline B & 2,4 & 0,063 & 2 & 2,1 & 2,1 & 0 & Sim \\
\hline $\mathrm{C}$ & 1,5 & 0,027 & 3 & 4,5 & 4,5 & 0 & Sim \\
\hline D & 2 & 0,027 & 4 & 6 & 6 & 0 & Sim \\
\hline E & 0,6 & 0,04 & 5 & 8 & 8 & 0 & Sim \\
\hline $\mathrm{F}$ & 3,4 & 0,063 & 6 & 6,6 & 13,4 & 6,8 & Não \\
\hline G & 1,5 & 0,027 & 7 & 11,4 & 11,4 & 0 & Sim \\
\hline $\mathrm{H}$ & 2 & 0,11 & 8 & 8,6 & 15,4 & 6,8 & Não \\
\hline 1 & 1 & 0,027 & 9 & 12,9 & 12,9 & 0 & Sim \\
\hline$J$ & 1,1 & 0,063 & 10 & 13,9 & 13,9 & 0 & Sim \\
\hline $\mathrm{L}$ & 1,5 & 0,027 & 11 & 15 & 15 & 0 & Sim \\
\hline M & 1,9 & 0,063 & 12 & 16,9 & 16,9 & 0 & Sim \\
\hline N & 1 & 0,027 & 13 & 17,9 & 17,9 & 0 & Sim \\
\hline
\end{tabular}

Fonte: dos autores (2017) 
Assim, o projeto teria seu caminho crítico, tempo e variância totais definidos, de acordo com a figura a seguir.

Figura 2: Rede PERT/CPM, com definição do tempo e caminho crítico do projeto

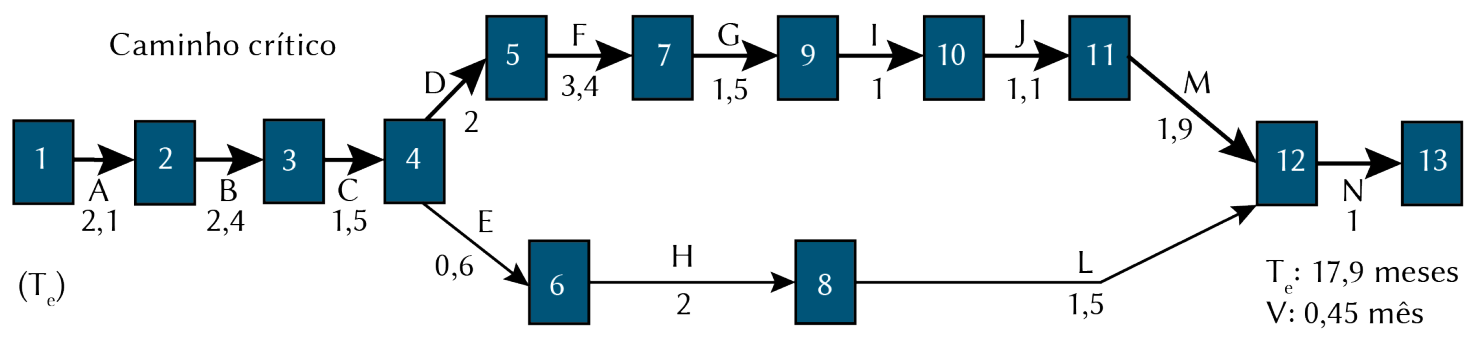

Fonte: dos autores (2017)

No esquema anterior, nota-se que o caminho crítico, ou seja, a sequência de eventos que possuem folga nula pertence à distribuição de atividades A-B-C-D-F-G-I-J-M-N (em destaque), as quais expressam o tempo total esperado para a execução do projeto (de 17,9 meses). Sabendo-se que o tempo fixado inicialmente para a conclusão do projeto pelos gestores é de 18 meses, seja qual for o cenário, aplica-se a fórmula estatística para o encontro do fator de probabilidade $(Z)$ na tabela de distribuição normal, a fim de identificar a probabilidade de viabilidade do projeto, conforme o especificado pela fórmula a seguir.

$$
\begin{gathered}
Z=\frac{\text { Tempo fixado }- \text { Tempo esperado }}{\sqrt{ } \text { Variância }} \\
\text { ou seja: } \\
Z=18-17,9 / \sqrt{ } 0,45 \longrightarrow Z=0,149,
\end{gathered}
$$

sendo que: 18 é o prazo fixado, 17,9 é o tempo total do projeto (percorrido no caminho crítico) e 0,45 é a variância total do projeto (soma das variâncias das atividades pertencentes ao caminho crítico).

$\mathrm{Na}$ avaliação por meio da tabela estatística de distribuição normal, para $Z=0,149$, a probabilidade de viabilidade do projeto $\mathbf{P}=\mathbf{5 5 , 5 7 \%}$.

Tabela 1: Distribuição normal com a identificação do fator de probabilidade $(Z=0,149)$

\begin{tabular}{cccccccccccc}
\hline $\mathbf{Z}$ & $\mathbf{0 , 0}$ & $\mathbf{0 , 0 1}$ & $\mathbf{0 , 0 2}$ & $\mathbf{0 , 0 3}$ & $\mathbf{0 , 0 4}$ & $\mathbf{0 , 0 5}$ & $\mathbf{0 , 0 6}$ & $\mathbf{0 , 0 7}$ & $\mathbf{0 , 0 8}$ & $\mathbf{0 , 0 9}$ \\
\hline $\mathbf{0 , 0}$ & 0,5000 & 0,5040 & 0,5080 & 0,5120 & 0,5160 & 0,5199 & 0,5239 & 0,5279 & 0,5319 & 0,5359 \\
$\mathbf{0 , 1}$ & 0,5398 & 0,5438 & 0,5478 & 0,5517 & $\mathbf{0 , 5 5 5 7}$ & 0,5596 & 0,5636 & 0,5675 & 0,5714 & 0,5753 \\
$\mathbf{0 , 2}$ & 0,5793 & 0,5832 & 0,5871 & 0,5910 & 0,5948 & 0,5987 & 0,6026 & 0,6064 & 0,6103 & 0,6141 \\
\hline $\mathbf{0 , 3}$ & 0,6179 & 0,6217 & 0,6255 & 0,6293 & 0,6331 & 0,6368 & 0,6406 & 0,6443 & 0,6480 & 0,6517 \\
\hline
\end{tabular}

Fonte: adaptado de Fonseca e Martins (1982) 
De acordo com Shimizu (1984), se $O<P<$ 0,25 , haveria pouca chance de viabilidade do projeto, apontando para desistência do gestor na aplicação de esforços e investimentos no projeto. Se $0,25<P<0,60$, o projeto teria boas condições de ser implantado e seria indicado o início de sua construção, como é o caso. Se $0,60<P<1$, o projeto teria ótimas condições de ser elaborado, mas isso poderia indicar excesso de recursos aplicados (como muita mão de obra que forneceu estimativas de tempo menores), os quais poderiam ser alocados em outros investimentos.

Para o custo esperado do projeto, por analogia, adota-se também a fórmula do tempo esperado, mas com os valores relativos ao custo previsto em cada cenário. Assim, o custo ficaria:

$\mathrm{T}_{\mathrm{e}}=\mathrm{C}_{\mathrm{e}}=\mathrm{O}+4 \mathrm{M}+\mathrm{P} / 6$

$C_{e}=1698+4(1857)+2130 / 6 \longrightarrow C_{e}=R \$ 1876,00$.

Dessa forma,o custo esperado na construção do projeto aponta para o valor de $\mathrm{R} \$ 1.876,00$, valor esse dentro do limite orçamentário disponível pela organização, que é de $\mathrm{R} \$ 1.900,00$.

Portanto, o exemplo supracitado mostra que o método PERT/CPM pode ser adotado no processo de gerenciamento de um projeto pelo setor público, por meio da avaliação e controle das atividades e prazos, em termos de custos, permitindo também a análise da viabilidade do projeto.

\subsection{Modelagem por programação linear}

Segundo Shamblin e Stevens Jr (1979), um modelo de programação matemática é especificado pela entrada dos termos (inputs). A Programação Linear (PL) é uma programação matemática em que a função-objetivo e as restrições assumem características lineares, tendo diversas aplicações no controle gerencial, como a administração de produção, análise de investimentos, logística empresarial, problemas de transportes, em síntese, problemas de utilização dos recursos disponíveis em que buscam utilização ótima dos mesmos, observando-se limitações impostas pelo processo produtivo ou pelo mercado. A Programação Linear pode ser descrita da seguinte forma (LACHTERMACHER, 2009, p. 26):

$$
\text { Otimizar } \quad z=f\left(x_{1} ; x_{2} ; x_{3} ; \ldots ; x_{n}\right)
$$

Sujeito a

$$
\left.\begin{array}{c}
g_{1}\left(x_{1} ; x_{2} ; x_{3} ; \ldots ; x_{n}\right) \\
g_{2}\left(x_{1} ; x_{2} ; x_{3} ; \ldots ; x_{n}\right) \\
\vdots \\
g_{m}\left(x_{1} ; x_{2} ; x_{3} ; \ldots ; x_{n}\right)
\end{array}\right\}=\left\{\begin{array}{c}
b_{1} \\
b_{2} \\
\vdots \\
b_{m}
\end{array}\right.
$$

Onde:

$f\left(x_{1} ; x_{2} ; x_{3} ; \ldots ; x_{n}\right)=c_{1} x_{1}+c_{2} x_{2}+c_{3} x_{3}+\ldots+c_{n} x_{n}$ $g_{i}\left(x_{1} ; x_{2} ; x_{3} ; \ldots ; x_{n}\right)=a_{i 1} x_{1}+a_{i 2} x_{2}+a_{i 3} x_{3}+\ldots+a_{n} x_{n}, i=1, \ldots, m$

$n$ - é o número de variáveis;

m-é o número de restrições;

$i$ é - o índice de uma determinada restrição.

Assim, a função a ser maximizada ou minimizada é denominada “função objetivo", ou seja, buscam encontrar a melhor distribuição possível de recursos entre diversas atividades ou tarefas para se atingir um valor ótimo conforme o objetivo estabelecido (LACHTERMACHER, 2009). Para a solução de problemas de Programação Linear, pode-se utilizaro Método Simplex, que é, segundo Caixeta Filho (2001), uma técnica para se encontrar algebricamente a solução 
ótima de um modelo que reúne recursos matemáticos, lógicos e computacionais, apoiados em modelos estruturados que permitem obter alternativas para tomada de decisões.

\subsubsection{Exemplo Prático}

O exemplo prático apresentado neste tópico refere-se à construção de um modelo para análise de custos no desenvolvimento de um projeto junto a uma empresa do ramo de construção civil.
Assim, têm se as atividades a serem desempenhadas e seus respectivos custos, quantidade de funcionários, quantidade de horas e custos homem-hora. Tendo em vista a determinação dos custos, as diversas atividades foram identificadas e orçadas.

Na Tabela 2, serão apresentados os custos dos funcionários para realizar as tarefas programadas. As demais fases foram calculadas de forma semelhante.

Tabela 2: Custo por atividade obtido junto à empresa em estudo

\begin{tabular}{cccc}
\hline FUNCIONÁRIO & CARGO & VALOR/HORA (R\$) & $\begin{array}{c}\text { DISPONIBILIDADE } \\
\text { (HORAS) }\end{array}$ \\
\hline $\mathrm{f} 1$ & $\mathrm{C} 1$ & 25,00 & 100 \\
$\mathrm{f} 2$ & $\mathrm{C} 2$ & 50,00 & 100 \\
$\mathrm{f} 3$ & $\mathrm{C} 3$ & 45,00 & 400 \\
$\mathrm{f} 4$ & $\mathrm{C} 4$ & 60,00 & 500 \\
$\mathrm{f} 5$ & $\mathrm{C} 5$ & 25,00 & 100 \\
\hline
\end{tabular}

Fonte: adaptado de Moro (2011)

A Tabela 2 compõe a função objetivo adiante esclarecida. Além desses referenciais, foram considerados também os dados referentes à quantidade de funcionários por fases, subdividida em semanas e meses; tempo utilizado para realização do projeto, especificado no modelo a seguir. $\mathrm{O}$ modelo aplicado tem como objetivo apurar os custos relacionados à mão de obra, minimizados em fluxo de caixa e pagamentos a serem efetuados semanalmente.
$\mathrm{Na}$ Tabela 3, será considerada a disponibilidade dos funcionários em cada fase do Desenvolvimento de Sistemas. Estes dados serão utilizados no modelo matemático, que será simulado a seguir. 


\begin{tabular}{|c|c|c|c|c|c|}
\hline \multirow{2}{*}{ Fases } & \multicolumn{5}{|c|}{ Funcionários } \\
\hline & F1 & F2 & F3 & F4 & F5 \\
\hline Fase 1 & 1 & 0 & 0 & 0 & 1 \\
\hline Fase 2 & 0 & 1 & 1 & 1 & 0 \\
\hline Fase 3 & 0 & 1 & 1 & 0 & 0 \\
\hline Fase 4 & 0 & 0 & 0 & 1 & 0 \\
\hline
\end{tabular}

Fonte: adaptado de Moro (2011)

O modelo, a seguir,refere-se à minimização dos custos relacionados às atividades programadas a serem executadas.

\section{Função Objetivo:}

Minimizar $Z=25 t 11+25 t 12+25 t 13+25$ t $14+50$ t $21+50$ t $22+50$ t $23+50$ t $24+45$ $\mathrm{t} 31+45 \mathrm{t} 32+45 \mathrm{t} 33+45+\mathrm{t} 34+60 \mathrm{t} 41+60$ $\mathrm{t} 42+60 \mathrm{t} 43+60 \mathrm{t} 44+25 \mathrm{t} 51+25 \mathrm{t} 52+25$ $\mathrm{t} 53+25 \mathrm{t} 54$

\section{Sujeito a}
a) $1 \mathrm{t} 11+0 \mathrm{t} 21+0 \mathrm{t} 31+0 \mathrm{t} 41+1 \mathrm{t} 51=150$
b) $0 \mathrm{t} 12+1 \mathrm{t} 22+1 \mathrm{t} 32+1 \mathrm{t} 42+0 \mathrm{t} 52=200$

c) $0 \mathrm{t} 13+1 \mathrm{t} 23+1 \mathrm{t} 33+0 \mathrm{t} 43+0 \mathrm{t} 53=250$

d) $0 \mathrm{t} 14+0 \mathrm{t} 24+0 \mathrm{t} 34+1 \mathrm{t} 44+0 \mathrm{t} 54=400$

e) $\mathrm{t} 11+\mathrm{t} 12+\mathrm{t} 13+\mathrm{t} 14<=100$

f) $\mathrm{t} 21+\mathrm{t} 22+\mathrm{t} 23+\mathrm{t} 24<=100$

g) $\mathrm{t} 31+\mathrm{t} 32+\mathrm{t} 33+\mathrm{t} 34<=400$

h) $\mathrm{t} 41+\mathrm{t} 42+\mathrm{t} 43+\mathrm{t} 44<=500$

i) $\mathrm{t} 51+\mathrm{t} 52+\mathrm{t} 53+\mathrm{t} 54<=100$

Com a aplicação do software Lindo ${ }_{\circledast}$ foram obtidos os resultados, conforme a distribuição das fases e os valores respectivos dos custos do projeto, resumidos na Tabela 4 e Tabela 5. 
Tabela 4: Tempos de funcionários por fase extraídos da simulação

\begin{tabular}{|c|c|c|c|c|c|}
\hline \multirow{2}{*}{ Fases } & \multicolumn{5}{|c|}{ Tempo/Funcionários (fn) } \\
\hline & Tempo f1 & Tempo f2 & Tempo f3 & Tempo f4 & Tempo f5 \\
\hline Fase 1 & 100 & 0 & 0 & 0 & 50 \\
\hline Fase 2 & 0 & 0 & 200 & 0 & 0 \\
\hline Fase 3 & 0 & 50 & 200 & 0 & 0 \\
\hline Fase 4 & 0 & 0 & 0 & 400 & 0 \\
\hline totais & 100 & 50 & 400 & 400 & 50 \\
\hline & & Total Geral & & & 1.000 \\
\hline
\end{tabular}

Fonte: adaptado de Moro (2011)

Tabela 5: Custos de funcionários por fase, extraídos da simulação (em R\$)

\begin{tabular}{cccccc} 
& \multicolumn{5}{c}{ Custos/funcionários (f) } \\
Fases & $\mathbf{f 1}$ & $\mathbf{f 2}$ & $\mathbf{f 3}$ & $\mathbf{f 4}$ & $\mathbf{f 5}$ \\
& 2500 & 0 & 0 & 0 & 1250 \\
Fase 1 & 0 & 0 & 9000 & 0 & 0 \\
Fase 2 & 0 & 2500 & 9000 & 0 & 0 \\
Fase 3 & 0 & 0 & 0 & 24000 & 1250 \\
Fase 4 & 2500 & 2500 & 18000 & 24000 & $\mathrm{R} \$ 48.250,00$ \\
\hline
\end{tabular}

Fonte: adaptado de Moro (2011) 
Com os resultados obtidos por meio da simulação com o software LINDO, verifica-se que a Função Objetivo gerou um custo otimizado de $\mathrm{R} \$ 48.250,00$ (quarenta e oito mil duzentos e cinquenta reais) para o Desenvolvimento de Sistemas-Projeto com Tempo Limite de 1.000 horas para realização.

Este resultado representa o conjunto das atividades, separadas por fases, e participações dos diversos funcionários de várias categorias de profissionais, executando o projeto de desenvolvimento do sistema pelo valor de $\mathrm{R} \$$ $48.250,00$, sendo esta importância o valor minimizado dos custos de realização do referido projeto.

Considerando esta solução compatível com as expectativas da empresa de construção civil, as horas acima representam o tempo a ser alocado de acordo com o projeto previsto, com custos mínimos, podendo ser consideradas como informações para inclusão no planejamento global de recursos humanos necessários para a empresa.

\section{CONCLUSÃO}

A partir da análise dos referenciais analisados, conclui-se que este trabalho alcançou seus objetivos, de modo que apresentou duas modelagens analíticas baseadas em técnicas de PO, utilizáveis no controle de custos durante o gerenciamento de projetos em organizações de setores diferentes: uma voltada ao desenvolvimento de sistemas de TI e outra do ramo da construção civil.

Primeiramente, foi apresentada uma modelagem fundamentada no método PERT/CPM, que teve sua aplicação prática no estudo de caso na empresa de software TI, em que foi possível verificar nessa situação as atividades e eventos envolvidos num projeto, de modo a planejar sua execução com base em três cenários projetados - pessimista, otimista e mais provável - e assim gerir o cronograma e o orçamento com os custos do projeto. Através disso, analisou-se também a viabilidade do projeto por meio do encontro dos totais de tempo obtido e custo incorrido, sob a ótica dos valores fixados pela respectiva organização.
Numa segunda etapa, foi delineada uma modelagem baseada em programação linear, em que se buscou planejar a minimização no custo de desenvolvimento de um projeto da área de construção civil (função objetivo), analisando certas restrições definidas, que no exemplo empregado caracterizaram-se em função de variáveis de tempo e custo de mão de obra empreendidos no projeto.

Salienta-se que tais modelagens podem ser empregadas de acordo com a situação objetivada por qualquer organização na gestão de seus custos, em qualquer tipo projeto, desde projetos de modernização, de produção e operacional (conforme apresentado nos estudos de caso), bastando apenas buscar as variáveis e modelar o estudo de acordo com o método que se quer aplicar para a análise, isto é, construir a modelagem do método conforme as características do projeto.É importante ressaltar que os exemplos práticos apresentados têm apenas uma função didática, com intuito de facilitar a compreensão das técnicas propostas. 


\section{ANALYTICAL MODELS APPLIED TO COST CONTROL IN THE MANAGEMENT OF PRODUCTION AND OPERATIONS}

\begin{abstract}
Cost management in production processes is quite complex and requires the use of appropriate techniques of analysis and control. Therefore, this article aims to describe analytical modeling that can be adopted in different situations, for any organization, in order to control costs in the management of productiveprocesses. For this, after a theoretical analysis based on some of the main operationalresearch techniques, the modeling was built according to each preset hypothetical situation, which could be experienced by an organization. Firstly, a case study in which the built model was based on PERT / CPM network, for the analysis of the time schedule and cost budget of a project. In another situation, a mathematical model was developed, which used the technique of linear programming to meet the goal of reducing costs in a specific project. Thus, such modelling presented can be applied according to the situation objectified by the organization in the management of production costs, only searching for the variables and model the study according to the method that one wants to apply for analysis.
\end{abstract}

KEYWORDS: Project management; cost planning; operational research.

\section{REFERÊNCIAS}

ACKOFF, R.L.; SASIENI, M. W. Pesquisa Operacional. Vol. 4. Rio de Janeiro: Ed. Livros Técnicos e Científicos, 1974.

ÁVILA, M. PMBOK e gerenciamento de projetos. Disponível em: <https://goo.gl/ $\mathrm{EGauLM}>$. Acesso em: 31 maio 2011.

BACHEGA, S. J; ANTONIALLI, L. M. Planejamento com PERT/CPM: um caso prático em uma pequena empresa rural que atua na produção e processamento de tilápias. Anais da SOBER. 2004. Disponível em: <https://goo.gl/ FeXoxT $>$. Acesso em: 15 set. 2010.

CAIXETA FILHO, J.V. Pesquisa operacional. São Paulo: Atlas, 2001.

DINSMORE, P. C.; CAVALIERI, A.

Gerenciamento de Projetos. Rio de janeiro: Qualitymark, 2003.

LACHTERMACHER, G. Pesquisa Operacional na Tomada De Decisões. 4. ed. São Paulo/SP: Campus, 2009.

MONTEVECHI, J. A. Pesquisa Operacional. UNIFEI, 2006.

PERES FILHO, H. C. Método prático de controle de custos numa empresa de projetos. In: I Congresso Brasileiro de Gerenciamento de Projetos. Anais.... Florianópolis, 2006.

PROJECT MANAGEMENT INSTITUTE PMI. A Guide to the Project Management Body of Knowledge - PMBOK. 4. ed. Newtown Square: Project Management Institute, 2012.

POMPERMAYER, C. B.; LIMA, J. E. P. Gestão de custos. In: Faculdades Bom Jesus/FAE Business School. Finanças Empresariais (Coleção Gestão Empresarial), Curitiba, v. 4, p. 49-58, 2002. 


\section{SOBRE OS AUTORES}

RORATTO, R. Fatores de risco no gerenciamento de projetos no setor público. Anais do VII SEGeT, 2011.

SANTOS, N. C. Captação de recursos financeiros em organizações sem fins lucrativos: a utilização de indicadores de gestão para os doadores e beneficiários dos projetos sociais. Revista de Gestão da USP, v. 15, n. especial, p. 75 - 91. São Paulo: USP, 2008.

SHAMBLIN, J. E.; STEVENS Jr., G. Pesquisa Operacional: uma abordagem básica. São Paulo: Atlas, 1979.

\section{SHIMUZU, T. Pesquisa Operacional em} engenharia, economia e administração: modelos básicos e métodos computacionais. Rio de Janeiro: Guanabara Dois, 1984.

SILVA, P. G.; COSTA, S. R. R. Gerenciamento de projetos em instituições públicas: um estudo de caso. Anais do VII SEGeT, 2010.

SOTILLE, M. Curso de Capacitação em Gerência de Projetos. PMI-RS, 2003.

TERENCE, A. C. F.; ESCRIVÃO FILHO. E. Abordagem quantitativa, qualitativa e a utilização da pesquisa-ação nos estudos organizacionais. Anais do XXVI ENEGEP, 2006.

VERGARA, S. C. Projetos e relatórios de pesquisa em administração. São Paulo: Atlas, 2006.

WILLIAMS, H. P. Model building in mathematical programming. 3. ed. Chichester: Wiley \& Sons, 1993.

Data de recebimento: 20/09/2016

Data de aprovação: 04/08/2017

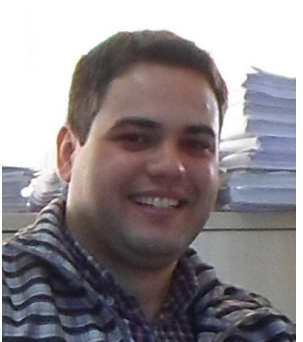

Rodrigo Roratto

Mestre em Engenharia de Produção (UFSM - 2012) e bacharel em Administração deEmpresas (UFSM-2008). No momento, atua como Professor Universitário da Faculdade Palotina de Santa Maria (FAPAS). Pesquisador técnico do CNPQ na UFSM na área de Gestão de Excelência no Setor Público, Perito Judicial (TJ/RS e JF/RS) e Administrador do quadro técnico-administrativo da Universidade Federal de Santa Maria (UFSM), com atuação no setor de logística de suprimentos. Possui também experiência em gerência da produção e operações. Ocupou diversos cargos de gestão nessa área, no segmento industrial do setor privado.

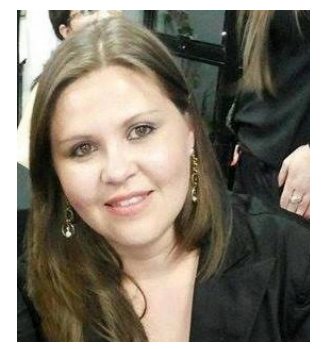

Edenilce Bittencourt Alves

Especialista em Controladoria e Auditoria (UNICESUMAR-2017)e bacharel em Administração em Empresas (FISMA - 2016). No momento, atua no ramo de negócios imobiliários, na área de gestão de projetos para o segmento e desenvolvimento de sistemas de gestão administrativa e operacional, com foco em modelagem de negócios através de tecnologias de informação, controle produtivo, gestão da qualidade e análise de performance empresarial. 


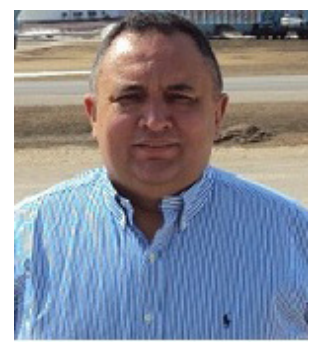

\section{Rodrigo Roratto}

Livre-docente pela

Universidade de São Paulo USP/ESALQ(2013), especialidade/disciplina: análise de decisões e gerenciamento de risco. Doutor em Ciências (Economia Aplicada) pela Universidade de São Paulo - USP/ESALQ (2010). Mestre e doutorado em Economia pela Escola de Pós-Graduação em Economia - EPGE/ FGV-RJ (1993), avaliado como o melhor departamento acadêmico de Economia da América Latina. Atividades de Pós-doutorado (pesquisador e professor-visitante), na USP/ESALQ/Departamento de Economia,Administraçãoe Sociologia(2010-2011), e no Instituto de Matemática, Estatística e Ciências da Computação - IMECC, da UNICAMP (2016). Professor livre-docente sob o regime de 40 h, DE, aprovado em concurso público, área de Finanças, na Universidade Federal de Alagoas - UFAL, a partir de 2015.

\section{$* * *$}

